\title{
A Multi-intelligent Agent System for Automatic Construction of Rule-based Expert System
}

\author{
Mohammed Abbas Kadhim \\ Department of Computer Science, College of Computer Science and IT, University of Al-Qadisiyah, Iraq \\ E-mail: moh_abbas74@yahoo.com \\ M. Afshar Alam and Harleen Kaur \\ Department of Computer Science, Hamdard University, New Delhi, India \\ E-mail: \{mailtoafshar, harleen_k1\}@rediffmail.com
}

\begin{abstract}
The main general purpose of this research is the automatic construction of rule-based expert system in diagnosis domain based on an expert system tool and a multi-intelligent agent system. The first goal is used an expert system tool (shell) which is called Diagnosis Domain Tool for Rule-based Expert System (DDTRES) [1]. The second goal is used a multi-intelligent agent architecture for knowledge extraction to elicit knowledge from its resources (domain experts, text documents, databases) for automatic construction of a knowledge base. That means, instead of using traditional methods for knowledge base construction, we used automatic way for that job. In order to achieve second objective, the following agents have been used: The Expert Mining Intelligent Agent (EMIA), The Text Mining Intelligent Agent (TMIA) [2], and The Multi-Intelligent Agent for Knowledge Discovery in Database (MIAKDD) [3]. We are aim to produce an effective final knowledge base by cooperation between EMIA, TMIA, and MIAKDD approaches and integrated with the diagnosis domain tool (DDTRES) to produce a complete rule-based expert system in diagnosis domain. We applied the captured rule-based expert system on heart diseases diagnosis, we found system performance is between a good and a very good range.
\end{abstract}

Index Terms - Intelligent agent system, Expert system shell, Rule-based expert system, Automatic construction of ES, Heart disease diagnosis.

\section{INTRODUCTION}

The basic characteristic that distinguishes our time today is the use of a computer system, which is one of the most essential indicators to point out the status of development in the life of society where intervention computers began in all areas of life such as administration, industry, trade, and other fields. In recent years, a new direction is beginning to build intelligent systems that depend essentially on the concept of Artificial Intelligence (AI). AI is sometimes referred to machine intelligence that is concerned with devising computer programs to make computer more intelligent. To reach this objective, we have two approaches; the first one include constructing the intelligent system in a method that is totally different from the way followed by a human. The second approach involve constructing a mechanisms and techniques similar to that applied by a human domain expert to solve complex problems. Expert system is one of the most common application of AI. It is a computer program that simulates the judgment and behavior of human domain expert or organization that has expert knowledge and experience in a specific particular field. Typically, such a system includes knowledge base containing accumulated experience and a set of rules for applying the knowledge base to each particular situation that is described to the program [4].

The other components of an expert system as well as the knowledge base are a user interface and inference engine. The process of acquiring knowledge from human experts and constructing a knowledge base in a particular domain is called knowledge engineering [5]. Fox defines knowledge engineering as "the engineering discipline that involves integrating knowledge into computer systems in order to solve complex problems normally requiring a high level of human expertise', [6].

The expert system tool is the software that is used for constructing expert systems. There are three software categories tools for development expert system: AI programming languages, expert system shells, and knowledge engineering languages [7]. In this research area, we focus on using a diagnosis domain tool (shell) for building rule-based expert system and constructing of its knowledge base by using a multi-intelligent agent system approach.

Human domain experts, natural language text documents, and databases are the main resources of knowledge for constructing expert systems. The extraction of that knowledge using classical methods is the bottleneck for building those systems. An expert system works on knowledge base that includes the problem solving knowledge eliciting from the above three resource. To represent knowledge base in knowledge-based system there are many method (production rules, frames, Bayesian networks, etc. The extraction of knowledge directly from domain experts, text documents, and databases allows for elicitation of knowledge easily and without intervention of knowledge 
engineers. The main idea of this research is eliciting interesting as well as vital knowledge from domain experts, text documents, and databases based on a multiintelligent agent system.

Intelligent agents or software intelligent agents are software entities that run a sequence of action on behalf of a human or another agent independently [8]. A MultiIntelligent Agent (MIA) is a collection of autonomous agents that interact with each other or their environments to perform one or more goals[9][10][11]. Recently, the use of intelligent agents have been applied in different applications such as intelligent agent user interface, independent agents, information recovery, and knowledge discovery and data mining. Multi-agent systems are a suitable solution to manage the dynamics and complexity of hybrid systems. A hybrid system is a system that uses combinations of artificial intelligence techniques[12]. Intelligent agents can be classified into two different categories: resident and mobile. Resident agents stay in the computer or system and perform their tasks there. Mobile agents can travel autonomously through different system architectures and platforms to fulfill their jobs [13][14]. In this research paper, we used a multi-intelligent agent architecture as a resident agents system.

In this paper, we will discuss the results of the previous three research papers and how they are combined to produce a complete rule-based expert system in diagnosis domain. We have captured the tool for construction rule-based expert system which is called DDTRES [1], as well as three knowledge bases were resulted from three systems: the EMIA, the TMIA [2], and the MIAKDD [3] using the production rules knowledge representation scheme to represent these knowledge bases. The verification and validation of a knowledge base in the expert system are important steps to ensure the quality of the rule-based expert system. The success of the expert system depends on the quality of knowledge in that knowledge base [15][16][17]. In the proposed system, the validation and verification (evaluation) for each knowledge base has been discussed in two previous research papers [2][3]. In the rest sections of the paper, we will discuss the final knowledge base, the knowledge base consistency and completeness, the complete rule-based expert system, and finally the system evaluation using heart diseases diagnosis.

\section{THE FINAL KNOWLEADGE BASE}

The above three knowledge bases which result from using the multi-intelligent agent must be combine to produce the final knowledge base that will be used with DDTRES to capture complete rule-based expert system in specific diagnosis domain.

After the combination process of the knowledge bases, the final knowledge base should be used an uniform production rules representation to avoid inconsistency of knowledge as in figure (1) which illustrates the combination process and the uniform production rules representation. The uniform production rules representation means collected all the contents of the knowledge bases in the final knowledge base using one form of production rules style. For example, the form of production rules in the EMIA's knowledge base is different from the form of the production rules produced by the MIAKDD's knowledge base.

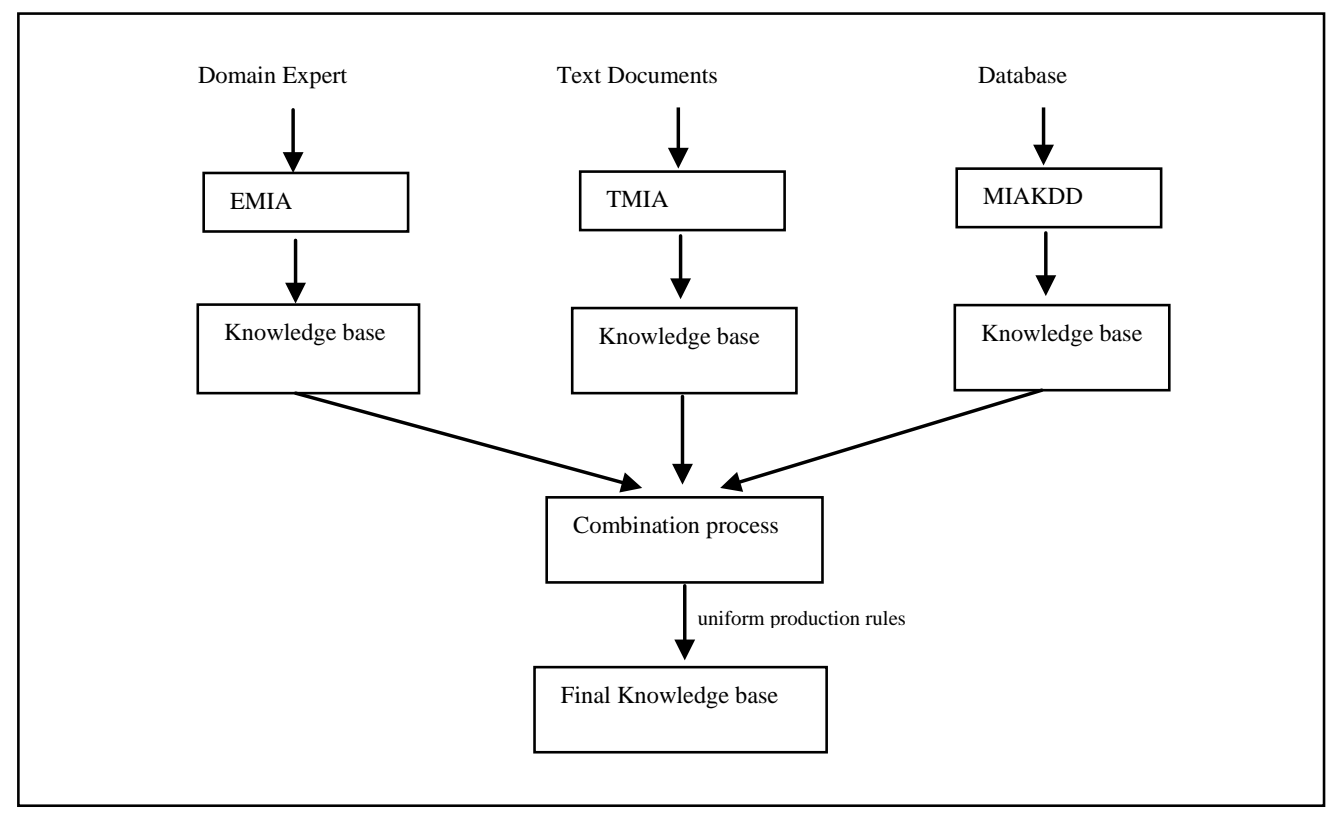

Fig.1. The combination process and uniform production rules scheme on the final knowledge base

When the uniform knowledge representation in the final knowledge base has been completed, or has at least at a sufficiently high level of accuracy, it is ready to be used. The completeness and consistency of the final 
knowledge base are the responsibilities of the combination process procedure. A complete and consistent knowledge base ensures that the expert system correctly represents the extracted knowledge from its resources, but does not involve that the system will produce correct results.

\section{KNOWLEDGE BASE COMPLETENESS}

The knowledge base completeness means that it is possible to reach all conclusions that can be drawn by the inference engine without any deficiency in its knowledge [18][19]. In the EMIA system, we produced an effective knowledge base through an interactive the system with more than one domain experts to guarantee the capture of a variety of knowledge from these different experts. The TMIA system produced the efficient and complete knowledge base because it deals with different text documents to extract knowledge under supervision of the domain expert.

The variety of knowledge comes also from the variety of resources for that knowledge (domain experts, text documents, and database ). In this way, we can obtain a complete knowledge for each knowledge base. The final knowledge base is already complete because it is results from the combination of the three completed knowledge bases.

\section{Kowledge BASE Consistancy}

A consistent knowledge base means that it is free of conflicting and redundant production rules. The conflicting rules mean that they will succeed in the same situation, but have contracting in conclusions. There are two types of redundancy: syntactic redundancy and semantic redundancy. In the syntactic redundancy, the rules have identical situations and identical conclusions, while in the semantic redundancy the rules have situations or conclusions with different syntax, but the meaning is same [19][20].

In the proposed system, the consistency of production rules for each knowledge base is the responsibility of the domain expert during the modification process procedures for each resource of knowledge, but the consistency of final knowledge base is responsibility of combination process procedure.

The consistency of the final knowledge base means that there is no conflicting between the newly added knowledge and the existing knowledge in the final knowledge base. For example in the proposed architecture, if the EMIA extracts a production rule for a specific situation from the domain expert and the TMIA extracts the same situation from text documents, this situation should be deleted by the combination process procedure when all knowledge bases combine in one final knowledge base. In this way, we guarantee there is no conflict between added new knowledge and saved knowledge in the final knowledge base.

\section{COMPLETE Rule-BASED EXPERT SySTEM}

When the constructing of knowledge base is the bottleneck of the rule-based expert system building. Therefore, the used multi-intelligent agent system focuses on constructing of knowledge base. In other words, the main task of the multi-agent architecture is to build the final knowledge base in rule-based expert system in diagnosis domain. The other components of rule-based expert system such as user interface, the inference engine, and explanation facility are constructed by the expert system tool (DDTRES) as in figure (2) which illustrates the combination between the expert system tool (DDTRES) and the final knowledge base which results from the multi-intelligent agent architecture. Now we captured the complete rule-based expert which can produce advices in the specific diagnosis domain (depend on the contents of the final knowledge base) such as medical diagnosis domain (back pain diseases, heart diseases...etc) or non-medical diagnosis domain (organic chemical, inorganic chemical... etc). The user interacts with the system through a user interface. In order to produce advice by the system, it displays symptoms as questions, the user answers for that questions by YES or NO, if the certain symptom appears or not as shown in figure (3-a) that illustrate an example for heart disease diagnosis.

In the end, depending on the user's answer, the name of the situation posted up on the screen as shown in figure(3-b) which illustrate the advice (conclusion) produced by the rule-based expert system. The system work like this: the system display first symptom (the symptom that has most frequent in all situations in working memory) if it has the positive answer then will keep only situations that have this symptom and eliminate all situations that don't have this symptom from working memory and vice-versa.

Then, display the second symptom (the symptom that has next most frequency for all situations in working memory) if it has positive or negative answer then will keep or eliminate the situations that have or don't have this symptom. Repeat this procedure until the system arrived at a particular situation (conclusion). The following algorithm illustrates the above description: 


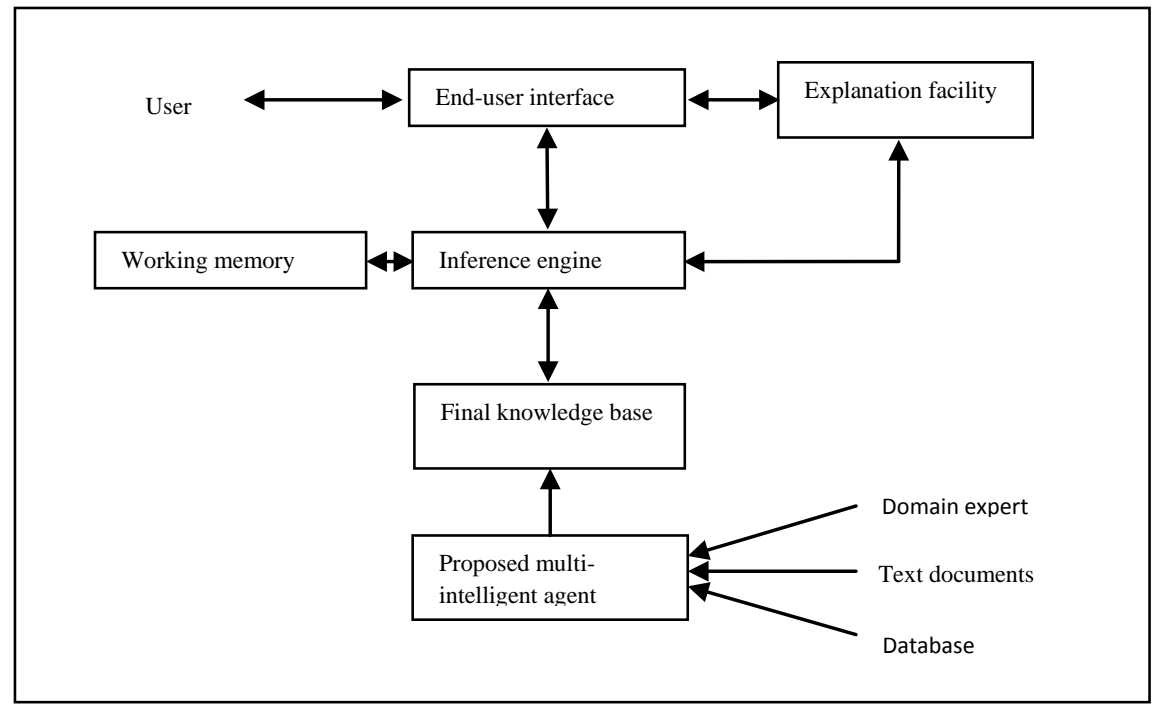

Fig.2. The complete rule-based expert system in a diagnosis domain

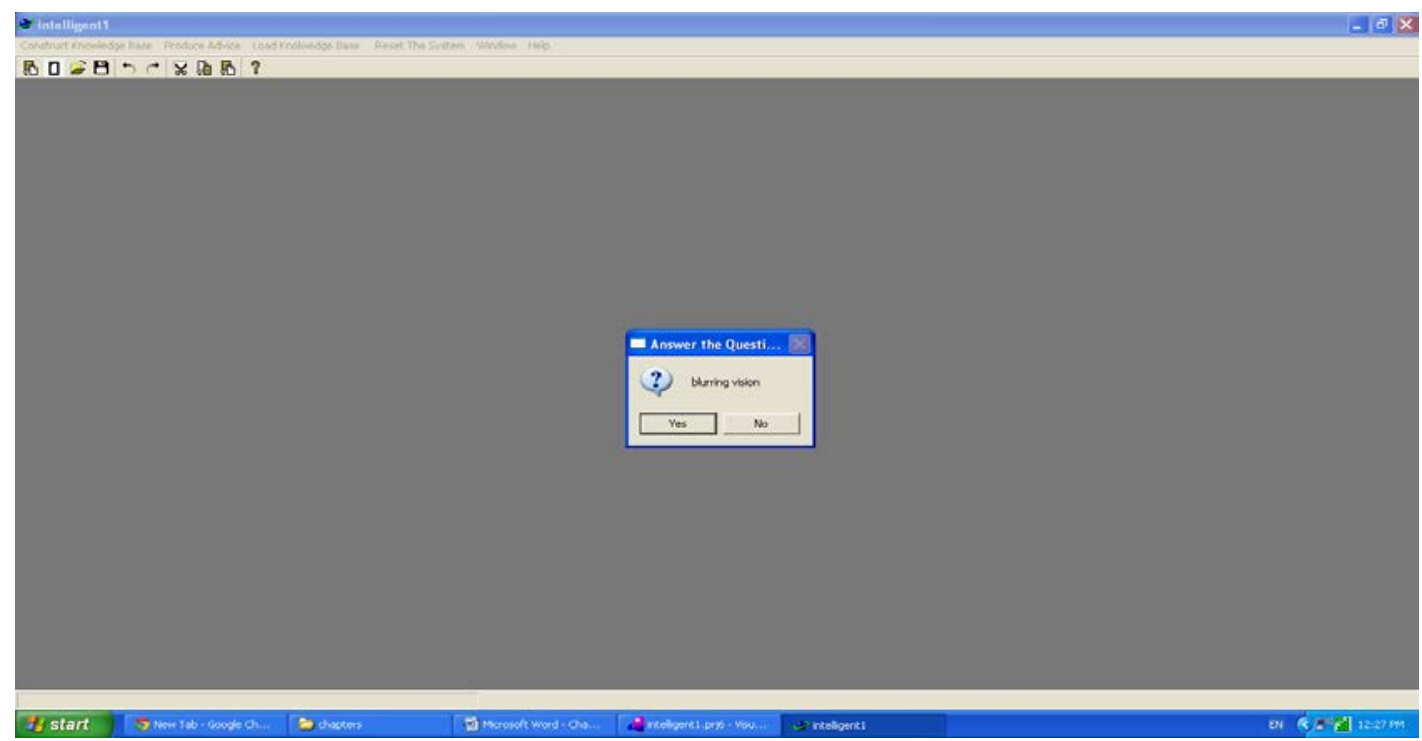

Fig.3-a. Interaction between user and the captured rule-based expert system

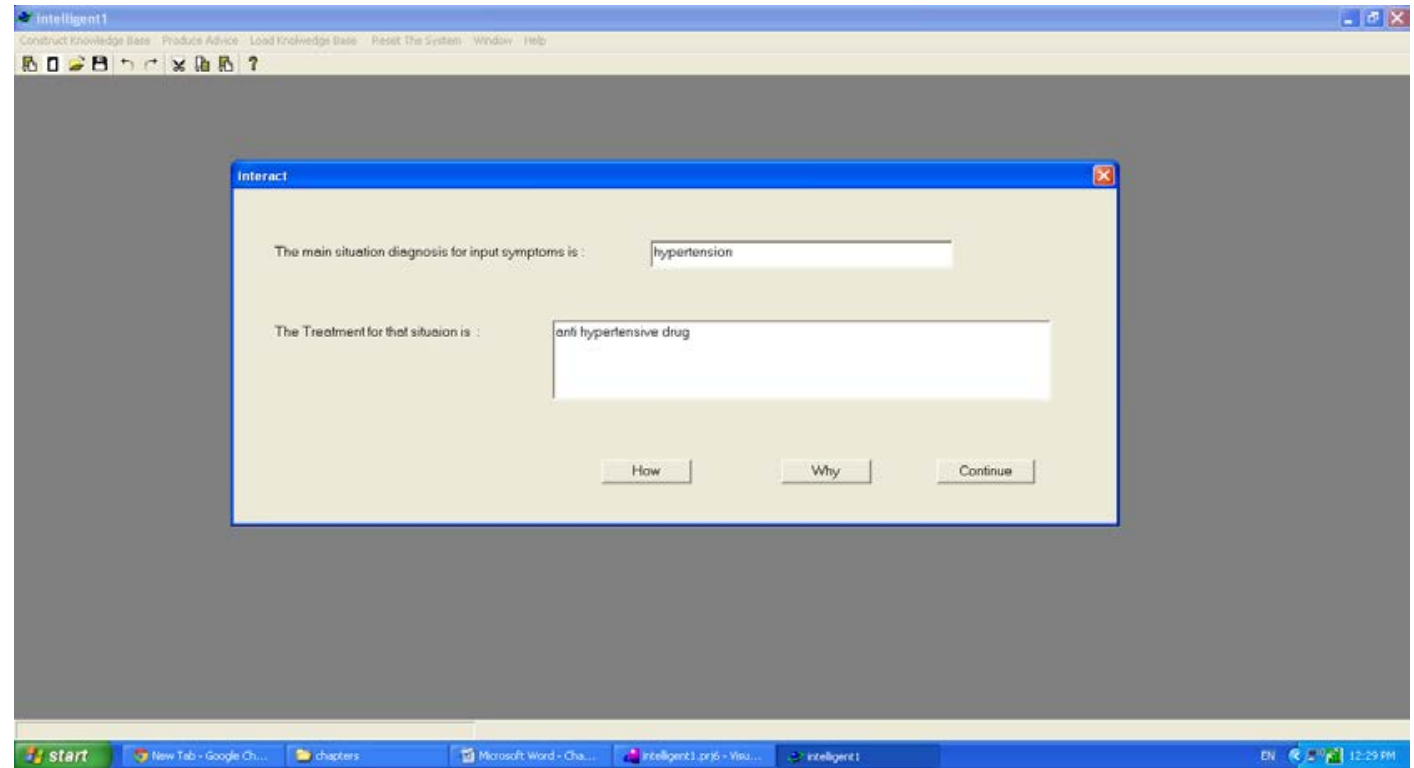

Fig.3-b. Produce advice by captured rule-based expert system 
Algorithm: Produced advice by the complete rule-based expert system

1. Kbase_File:=the knowledge base file

2. consult (Kbase_File)

//load all situations and its symptoms from knowledge base file to working memory using consult procedure

3. find the most frequent symptom Freq_Symptom

//find the most frequent symptom in all situations in working memory

4. display Freq_Symptom to answer 'YES' or 'NO' by user

5. if Answer='YES' then

6. save all situations that have Freq_Symptom

7. remove all situations that don't have Freq_Symptom

// keep all situations that have this symptom and remove all situations that don't have this symptom in/from working memory

8. $\quad$ else

9. remove all situations that have Freq_Symptom

10. remove Freq_Symptom from all saved situations //prepare to find next most frequent symptom in all saved situations that already have Freq_Symptom

11. if working memory contain only one situation then

12. display this situation as an advice (decision) to the user

13. else

14. goto step 3

//back to find next most frequent symptoms in contain of working memory

15. end

\section{Rule-BASED EXPERT EVALUATION}

The evaluation process of an expert system is performed in two steps: verification and validation. Verification refers to building the system right, while validation refers to building the right system [21]. Validation and verification are fundamental steps in all systems development to ensure the quality of these systems [20]. Many expert systems were validated, verified, and tested using case studies, the results of which were conducted by the knowledge engineer [22][23]. These processes involve reading and testing the knowledge base looking for semantic and syntactic errors and searching for incompatible between the output of the expert system and the expertise. This leads to being time consuming, error prone, program debugging, and does not guarantee finding all lacks in the knowledge base especially for large systems [19].

The heart diseases diagnosis domain has been used in the complete rule-based expert system as a case study. To evaluate the captured rule-based expert system must be achieved the verification and validation processes. In the verification process, it is determined the possible errors in the system and ensure that system achieved as proposed or the system implements its specifications correctly. This process can be accomplished by carry out the system many times. The result produced by the system was verified by domain experts. In the verification phase, the captured expert system was tested and verified by three domain experts in the above domain field. We selected three heart diseases as test cases and displayed the symptoms of these cases to the expert system and the domain experts independently. Both of them reached the same recommendations in three tested cases. It is also by tracing the execution of those tested cases, we found the system is working properly.

The second step of the evaluation process is the system validation which includes methodology validation by the domain experts or live testing, or prove the consultation that produced by the system with an acceptable level of precision. The validation process in the captured expert system was conducted by three domain experts. We were asked them for carrying out the captured system and full a validation form by evaluation marks which reflects their opinion with the system's performance. The validation form consists of the following criteria: friendliness of the end-user interface and clarity of the questions, completeness of the knowledge base, correctness of the system recommendations, benefit of the system results, and educational relevance [21][24]. The evaluation marks for the above criteria are qualitative grades (unacceptable, acceptable, good, very good and excellent) based on the confidence of the domain experts with the above criteria and the system's performance. Table 1 illustrates the evaluation mark scores after convert them to quantitative grades (Unacceptable $=0$, Acceptable $=10$, Good=20, Very $\operatorname{good}=30$, Excellent $=40$ ).

Table 1. The evaluation mark scores produced by domain experts for the captured expert system

\begin{tabular}{|l|c|c|c|c|}
\hline The evaluation criteria & $\begin{array}{c}\text { Domain } \\
\text { expert 1 }\end{array}$ & $\begin{array}{c}\text { Domain } \\
\text { expert 2 }\end{array}$ & $\begin{array}{c}\text { Domain } \\
\text { expert 3 }\end{array}$ & Average \\
\hline User friendliness \& questions clarity & 20 & 30 & 10 & 20 \\
\hline Completeness of the knowledge base & 30 & 40 & 20 & 30 \\
\hline Correctness of the recommendations & 30 & 30 & 10 & 23.3 \\
\hline Benefit of the system & 30 & 30 & 20 & 26.6 \\
\hline Education relevance & 40 & 30 & 20 & 30 \\
\hline
\end{tabular}

produced by the domain experts and figure (4) illustrates the graphical representation of that evaluation marks. The average column in the above table shows us the overall system performance is between a good and a very good range, with 20 grade for friendliness of the end-user interface and clarity of the questions asked, 30 grade for 
the completeness of the knowledge base, 23.3 grade for the correctness of the system recommendations and conclusions, 26.6 grade for the benefit of the system results, and finally 30 grade for the educational relevance.

\section{CONCLUSIONS}

In this research paper, we have captured a rule-based expert system in a diagnosis domain which results from a combination of two systems. In the first one, we used expert system tool (shell) in a diagnosis domain which is called DDTRES. In the second system, we used a multiintelligent agent for constructing a final knowledge base automatically in a specific diagnosis domain. The automatic knowledge acquisition approaches can reduce the required time for constructing of the knowledge base. That means, instead of using traditional methods to extract knowledge we can use the multi-intelligent agent system for that job automatically with minimum time and cost.

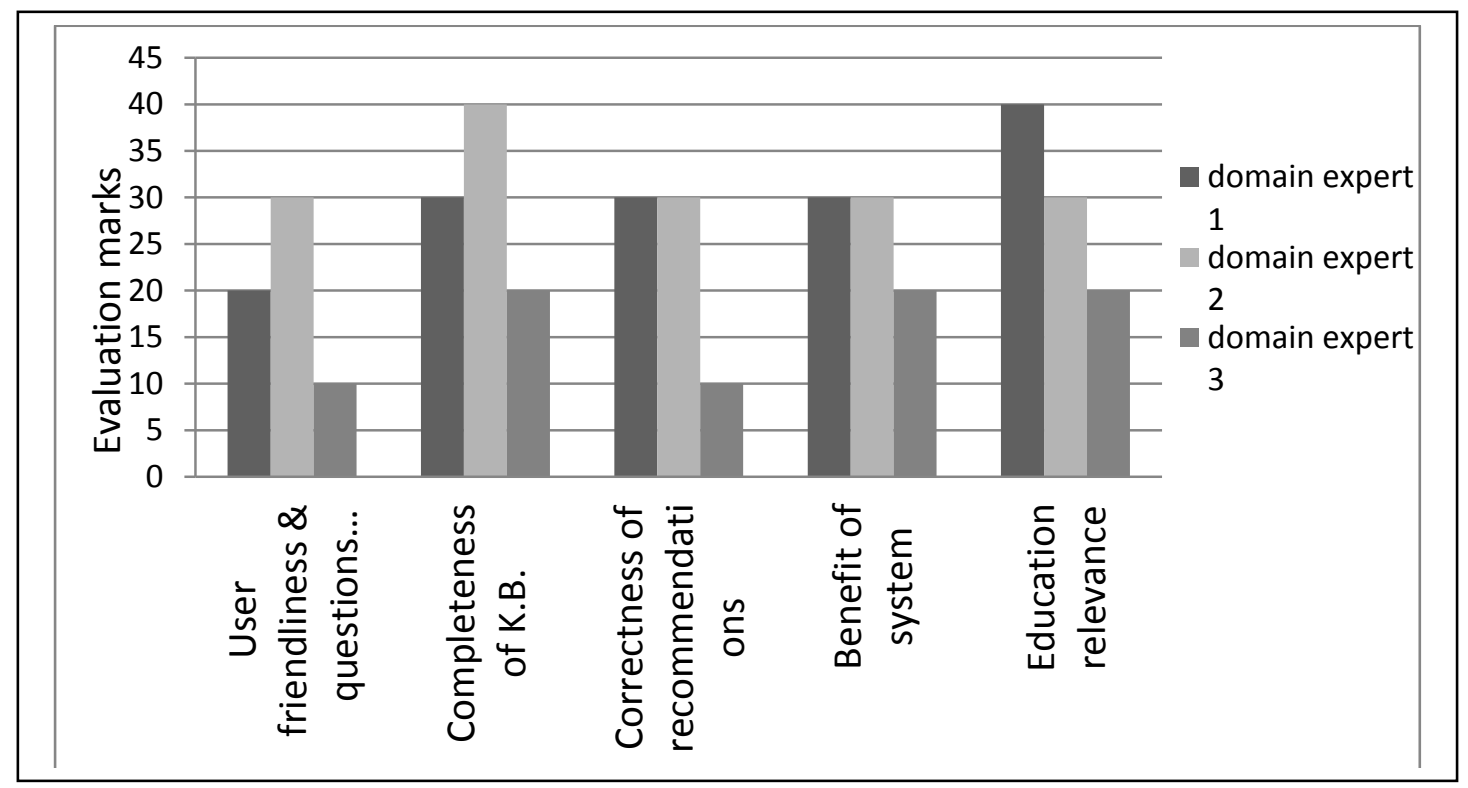

Fig.4. Graphical representation of the system evaluation

The complete rule-based expert system has been evaluated to prove the system performance using case study tests by the domain experts based on a set of criteria. The result of that evaluation is between a good and a very good range. That means, the performance of the system gives correct, effect, and reliable recommendations which are similar to the domain experts' recommendations under same conditions.

Finally in this paper, we produced a rule-based expert system automatically without intervention of the knowledge engineer in a specific diagnosis domain. Furthermore, we discussed a general model for automatic constructing of rule-based expert system based on the expert system shell and the resident multi-intelligent agent system.

\section{ACKNOWLEDGMENT}

The author expresses his gratitude to Dr. Rajiv Agarwal and Dr. Rajiv Bajaj in Batra Hospital New Delhi, and Dr. Kamaljit Singh in Kamal Medicare Clinic for their role in the system evaluation and knowledge acquisition processes.

\section{REFERENCES}

[1] Kadhim, M. A., Alam M. A., and Kau H. ,” Design and implementation of Intelligent Agent and Diagnosis Domain Tool for Rule-based Expert System“, International Conference on Machine Intelligence Research and Advancement (ICMIRA, 21 $1^{\text {st }}-23^{\text {rd }}$ Dec 2013), conference proceedings by IEEE Xplore, pp. 619622, 2013.

[2] Kadhim, M. A., Alam, M.A., and Kaur, H., “A Multiintelligent Agent Architecture for Knowledge Extraction: Novel Approaches for Automatic Production Rules Extraction", International Journal of Multimedia and Ubiquitous Engineering, Vol. 9, No. 2, pp.95-114, 2014.

[3] Kadhim, M. A, M. Afshar Alam, and Harleen Kaur,”A Multi-Intelligent Agent for Knowledge Discovery in Database (MIAKDD): Cooperative Approach with Domain Expert for Rules Extraction”, International Conference on Intelligent Computing, Intelligent Computing Methodologies, LNCS, Vol. 8589, pp 602614, 2014.

[4] Rich, Elaine, "Artificial Intelligence", McGraw-Hill , $1^{\text {st }}$ edition , 1983.

[5] Turban, E., Aronson, J.E., Liang, T., \& Sharda, R., "Decision Support and Business Intelligence Systems", Published by Dorling Kindersley (India) Pvt. Ltd., $8^{\text {th }}$ Edition, 2009.

[6] Fox, J., "Formalizing knowledge and expertise: where have we been and where are we going?”, The Knowledge Engineering Review, Vol. 26, Issue 1, pp. 5-10, 2011.

[7] Eldrandaly, K., ”An Intelligent MCDM Approach for Selection the Suitable Expert System Building Tool”, The 
International Arab Journal of Information Technology, Vol. 4, No. 4, pp. 365-371, 2007.

[8] Duan, Y., Ong, V. K., Xu, M., \& Mathews, B., "Supporting decision making process with "ideal" software agents - What do business executives want ?", Expert System with Applications, Vol. 39, Issue 5, pp. 5534-5547, 2012.

[9] Ropero, J., Gomes, A., Carrasco, A., \& Leon C., “A Fuzzy Logic intelligent for Information Extraction: Introducing a new Fuzzy Logic-based term weighting scheme" Expert Systems with Applications, Vol. 39, Issue 4, 2012, pp. 4567-4581, 2012.

[10] Ralha, C. G., \& Silva, C. V. S., "A Multi-agent data mining system for cartel detection in Brazilian government procurement”, Expert Systems with Applications, Vol. 39, Issue 14, pp. 11642-11656, 2012.

[11] Kadhim, M. A., Alam, M. A. , " To Developed Tool, an Intelligent Agent for Automatic Knowledge Acquisition In Rule-based Expert System”, International Journal of Computer Applications(IJCA), Vol. 42, No. 9, pp. 46-50, 2012.

[12] Kazik, O. and Roman Neruda, "Ontological Modeling of Meta Learning Multi-Agent systems in OWL-DL", IAENG International Journal of Computer Science, Vol. 39, Issue 4, pp. 357-362, 2012.

[13] Kim, D., Kim, C., \& Rim, K., "Modeling and Design of Intelligent Agent System", International Journal of Control, Automation, and Systems, Vol. 1,No. 2, pp. 257261,2003

[14] Turban, E., "Software (Intelligent) Agents", www.scribd.com/doc/56875420/Turban-Online-TechApp C, Technical Appendix C, (1999), (accessed 7 November 2015).

[15] Abraham, A., "Rule-based expert systems- Handbook of Measuring System Design”, John Wiley \& Sons, 2005.

[16] Kaur, H., Wasan, S K., Al-Hegami. A. S., Bhatnagar V, A Unified Approach for Discovery of Interesting Association Rules in Medical Databases, Advances in Data Mining, Lecture Notes in Artificial Intelligence series, 4065:53-63, Springer-Verlag, Heidelberg, 2006.

[17] Kaur H, Chauhan R, Data Mining Cluster analysis on the influence of health factors in Casemix data, BMC Journal of Health Services Research, June, 2012.

[18] Turban, E., R. Sharda and D. Delen, "Decision support and business intelligence systems", 9th edition, Prentice International Hall, USA, 2011.

[19] Fogelqvist, P., "Verification of completeness and consistency in knowledge-based systems", Master Thesis, Department of Informatics and Media, Uppsala University, Sweden 2011.

[20] Gonzalez, A.J. and D.D. Dankel., "The engineering of knowledge-based systems - theory and practice", Englewood Cliffs, Prentice-Hall, 1993.

[21] Devraj, Renu Jain, "PulsExpert: An expert system for the diagnosis and control of diseases in pulse crops", Expert Systems with Applications, Vol. 38, Issue 9, pp.1146311471, 2011.

[22] Jayawardhana, L. C., Aruna M., Ajith D., Malik R., Sumith P., and Indrika A., "BESTCOMP: expert system for Sri Lankan solid waste composting”, Expert Systems with Applications, Vol. 24, Issue 3, pp. 281-286, 2003.

[23] Kaur, H., Chauhan. R., M Afshar, Alam., S. Aljunid., and M, Salleh, SpaGRID: A Spatial Grid Framework for High Dimensional Medical Databases, Hybrid Artificial Intelligence Systems, Lecture Notes in Computer Science,Vol. 7208, pp. 690-704,2012.

[24] Gonzalez-Diaz, L., P. Martínez-Jimenez, F. Bastida , and
J.L. Gonzalez-Andujar, "Expert system for integrated plant protection in pepper (Capsicum annuun L.)”, Expert Systems with Applications, Vol. 36, Issue 5, pp. 89758979, 2009.

\section{Authors' Profiles}

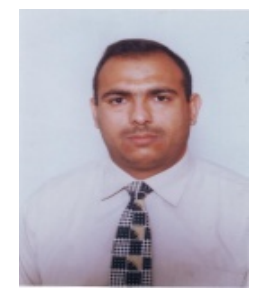

Mohammed Abbas Kadhim is Assistant Professor in College of Computer Science and IT, University of Al-Qadisiyah, Iraq. He gained his Ph.D. in Computer Science Dept., Hamdard University, New Delhi, India on the topic of a multi-intelligent agent for automatic construction of expert system. He received his B.Sc. and M.Sc. degree in Computer Science from Babylon University, Babylon, Iraq in 1996 and 1999 respectively. He has published many research papers in Artificial Intelligence field. His research interest includes expert systems, intelligent agents, text mining, data mining, neural network, and genetic algorithm.

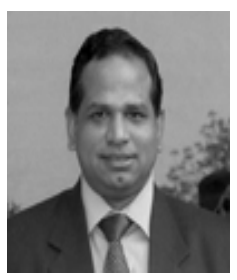

M. Afshar Alam is a Professor in Computer Science, he was Head of Computer Science Department, Faculty of Management and Information Technology, at the Hamdard University, New Delhi, India. In 1997-2000, he founded the Department of Computer Science, Hamdard University. He was also founder of Computer Centre at Hamdard University. He received his Master degree in Computer Science from the Aligarh Muslim University, Aligarh and Ph.D. from Jamia Millia Islamia University, New Delhi. His research interests include Fuzzy logic, Software engineering and Bioinformatics. He is the author of a book on Software re-engineering and over 50 publications in International/ National journals, conference and chapter in an edited book. He is a member of expert committee AICTE, DST, UGC and Ministry of Human Resource Development (MHRD), New Delhi, India.

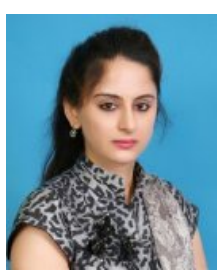

Harleen Kaur gained her Ph.D. in Computer Science from Jamia Millia Islamia University, New Delhi, India on the topic of Applications of Data Mining techniques in Health care Management. She graduated from the University of Delhi, New Delhi. She has previously served as a Lecturer in Computer Science, University of Delhi. Currently, she is an Assistant Professor at the Department of Computer Science, Hamdard University. She has published numerous research articles in refereed international journals and conference proceedings and chapters in an edited book. She is a member of several international bodies. Her main research interests are in the fields of Data analysis with applications to medical databases, Medical decision making, Fuzzy logic, Information Retrieval, Bayesian networks and visualization. 